\title{
Sounds good: the bright future of clinical alarm
}

\section{management initiatives}

\author{
Halley Ruppel, ${ }^{1}$ Christopher P Bonafide ${ }^{2}$
}

'Division of Research, Kaiser Permanente Northern California Oakland, California, USA ${ }^{2}$ Section of Pediatric Hospital Medicine, The Children's Hospital of Philadelphia, Philadelphia, Pennsylvania, USA

\section{Correspondence to} Dr Halley Ruppel, Division of Research, Kaiser Permanente Northern California, Oakland, California, USA;

Halley.W.Ruppel@kp.org

Accepted 12 February 2020 Published Online First

21 February 2020

\section{Sinked}

- http://dx.doi.org/10.1136/ bmjqs-2019-010368

\section{Check for updates}

(C) Author(s) (or their employer(s)) 2020. No commercial re-use. See rights and permissions. Published by BMJ.

To cite: Ruppel $\mathrm{H}$, Bonafide CP. BMJ Qual Saf 2020;29:701-703.
For decades, those working in hospitals normalised the incessant alarms from medical devices as a necessary, almost comforting, reality of a high tech industry. While nurses drowned in excessive, frequently uninformative alarms, other members of the healthcare team often paid little attention. Fortunately, times are changing and managing alarm fatigue is now a key patient safety priority in acute care environments. ${ }^{1}$

Adverse patient events from alarm fatigue, particularly related to excessive physiological monitor alarms, have received widespread attention over the last decade, including from the news media. ${ }^{2-5}$ In the USA, hospitals redoubled alarm safety efforts following the 2013 Joint Commission Sentinel Event Alert and subsequent National Patient Safety Goals on alarm safety. ${ }^{126}$ We are now beginning to understand how to reduce excessive non-actionable alarms (including invalid alarms as well as those that are valid but not actionable or informative), ${ }^{78}$ better manage alarm notifications and ultimately improve patient safety. Alarm data are readily available and measuring alarm response time during patient care is possible. ${ }^{79}$ Yet we have few high-quality reports describing clear improvement to clinical alarm burden, and most published interventions are of limited scope, duration or both. ${ }^{10} 11$ To demonstrate value in alarm quality improvement (QI) efforts moving forward, we need more rigorous evidence for interventions and more meaningful outcome measures.

In this issue of BMJ Quality and Safety, Pater $e t a l^{12}$ report the results of a comprehensive multidisciplinary alarm management QI project executed over 31/2 years in a 17-bed paediatric acute care cardiology unit. The primary project goal was to reduce alarm notifications from continuous bedside monitoring. Although limited to a single unit, the project is an important contribution to the scant literature on alarm management in paediatric settings for three reasons. First, the initiative lasted longer than most that have been reported, which allowed for tailoring of alarm interventions to the needs of the unit and patient population and measuring the impacts and sustainability over time. Second, the scope of the intervention bundle encompassed a wide variety of changes including adoption of a smartphone notification system; addition of time delays between when alarm thresholds are violated and when an alarm notification is issued; implementation of an alarm notification escalation algorithm after a certain amount of time in alarm threshold violation; deactivation of numerous technical alarms (such as respiratory lead detachment); monitoring of electrode lead replacement every 24 hours; and discussion of alarm parameters on daily rounds. Third, the authors introduced a novel strategy for reducing the stress that alarms may cause patients and families by deactivating inroom alarm audio, although no outcomes were reported attributable directly to this component of the intervention.

This project constitutes an important contribution to the published literature; however, Pater et al faced two challenges that are ubiquitous in the field of clinical alarm management: (1) Identification of meaningful outcome measures and (2) Lack of high-quality evidence for most interventions. With regards to the first challenge, the primary outcome measure used in the study comprised 'initial alarm notifications', defined as the first notification of a monitor alarm delivered to the nurse's mobile device. Although initial alarm notifications declined by $68 \%$ 
following the intervention, these notifications accounted for only about half of all alarm notifications. The other half included second and third notifications for alarms exceeding specified delay thresholds, which were sent both to the mobile device of the primary nurse and to 'buddy' nurses, potentially increasing alarm burden. On the other hand, eliminating inroom audible alarms may have reduced the perceived alarm burden for nurses compared with having both bedside and mobile device notifications. Determining the true benefit of a reduction in a subset of alarms presents complex challenges.

Alarm frequency is the most commonly used outcome measure in alarm research and QI projects, but reduction in alarms does not necessarily indicate improved patient safety or a highly functional alarm management system. Alarm reduction could easily be achieved in an undesirable way by simply turning off alarms. Unfortunately, most studies have not been powered to statistically evaluate improvements in patient safety. (Pater et al did monitor patient safety balancing measures, which remained stable after intervention implementation). To assess change in nurses' perceptions of alarm frequency, Pater et al conducted a prepost survey, which despite the small sample size $(n=38$ preintervention and $n=25$ postintervention) managed to show improvement, with the percentage of nurses agreeing they could respond to alarms appropriately and quickly increasing from 32\% to $76 \%(\mathrm{p}<0.001)$. That said, this survey was not a validated measure of alarm fatigue. In fact, we currently have no widely accepted, validated tool for assessing alarm fatigue. ${ }^{11}$

As we look towards future evaluations of alarm management strategies, the focus needs to shift away from simply reducing the frequency of alarms to more meaningful outcome metrics. In addition to alarm rates, outcomes such as response time to actual patient alarms $^{79}$ or to simulated alarms injected into real patient care environments ${ }^{13}$ may be better indicators of whether the entire alarm response system is functioning correctly. Larger, multisite studies are needed to assess patient outcomes.

In addition to meaningful outcome measures, the second challenge for alarm QI projects is the lack of good evidence for alarm management interventions. Most alarm reduction interventions have not been systematically evaluated at all or only in small studies without a control group. ${ }^{10}{ }^{11}$ As a result, alarm management projects tend to involve complex and costly bundles of interventions of uncertain benefit. The cost of these interventions is due in part to the growing industry of technology solutions for alarm management. Some institutions have also made massive investments in personnel, such as monitor 'watchers' to help nurses identify actionable alarms, for which there is also little evidence. $^{14}$

Future alarm management QI initiatives will benefit from a higher quality evidence base for the growing list of potential alarm management interventions. Pragmatic trials that leverage meaningful outcome measures to assess alarm interventions are warranted. In addition, we need to evaluate interventions that address the full spectrum of the alarm management system. Most alarm management interventions to date have focused primarily on filtering out non-actionable alarms. Far less emphasis has been placed on ensuring that the nurse receiving the notification is available to respond to the alarm, a prime opportunity for future work.

Even if alarms are actionable, we know that nurses may not always respond quickly for a variety of reasons. ${ }^{75-17}$ Factors like insufficient staffing, high severity of illness on the unit and unbalanced nursing skill mix all likely contribute to inadequate alarm response. In critical care, nurses have reported that the nature of their work requires that they function as a team to respond to one another's alarms. ${ }^{15}$ Although not ideal, nurses have developed heuristics based on factors like family presence at the bedside to help them prioritise alarm response in hectic work environments. ${ }^{76}$ Emphasising outcomes like faster alarm response time without addressing systems factors risks trading one patient safety problem for another. We do not want to engender more frequent interruptions of high-risk activities, like medication administration, ${ }^{18} 19$ because nurses feel compelled to respond more quickly to alarms.

The robust QI initiative carried out by Pater et al reflects the type of thoughtful approach needed to implement and tailor alarm management interventions for a particular unit, demonstrating a generalisable process for others to emulate. Ultimately, every alarm offers a potential benefit (opportunity to rescue a patient) and comes with a potential cost (eg, increased alarm fatigue, interruptions of other activities). This trade-off needs to be optimised in the context of the individual unit, accounting for the unit-specific and systems factors that influence the cost of each additional alarm, including non-actionable alarm rates, unit layout, severity of illness and nurse staffing. ${ }^{1720}$ With more robust outcome measures and more evidence to support interventions, we can increase the value of alarm QI initiatives and accelerate progress towards optimising alarm management systems.

Acknowledgements We thank Charles McCulloch, PhD (University of California, San Francisco) for comments on an early draft.

Funding HR was supported by NIH K12HD052163. CPB's effort on this manuscript was supported by an Agency for Healthcare Research and Quality Patient Safety Learning Laboratory award (R18HS026620). The content is solely the responsibility of the authors and does not necessarily represent the official views of the Agency for Healthcare Research and Quality. CPB discloses additional grant funding from other federal sources including awards from the National Institutes of Health and the National Science Foundation.

Competing interests None declared.

Patient consent for publication Not required.

Provenance and peer review Commissioned; internally peer reviewed. 


\section{REFERENCES}

1 The Joint Commission. Hospital National Patient Safety Goals, 2020. Available: https://www.jointcommission.org/-/media/tjc/ documents/standards/national-patient-safety-goals/2020-hapnpsg-goals-final.pdf [Accessed 30 Jan 2020].

2 The Joint Commission. Sentinel event alert: medical device alarm safety in hospitals, 2013. Available: http://www. jointcommission.org/assets/1/18/SEA_50_alarms_4_5_13_ FINAL1.PDF [Accessed 30 Jan 2020].

3 Kowalczyk L. State reports 11 patient deaths linked to alarm fatigue in Massachusetts. Boston Globe, 2011www. bostonglobe.com/metro/2011/12/29/state-reports-detailpatient-deaths-linked-alarm-fatigue-massachusetts/C8y3itRv d4WiGnR40sVHxN/story.html [Accessed 10 Feb 2020].

4 Kowalczyk L. No easy solutions for alarm fatigue. Boston Globe, 2011. Available: www.boston.com/lifestyle/health/ articles/2011/02/14/no_easy_solutions_for_alarm_fatigue/ [Accessed 10 Feb 2020].

5 Kowalczyk L. Groups target alarm fatigue at hospitals. Boston Globe, 2011. Available: www.boston.com/lifestyle/health/ articles/2011/04/18/groups_target_alarm_fatigue_at_hospitals/ [Accessed 10 Feb 2020].

6 Joint Commission on Accreditation of Healthcare Organizations. The Joint Commission announces 2014 National Patient Safety Goal. Jt Comm Perspect 2013;33:3-4.

7 Bonafide CP, Localio AR, Holmes JH, et al. Video analysis of factors associated with response time to physiologic monitor alarms in a children's Hospital. JAMA Pediatr 2017;171:524-31.

8 Rayo MF, Moffatt-Bruce SD. Alarm system management: evidence-based guidance encouraging direct measurement of informativeness to improve alarm response. BMJ Qual Saf 2015;24:282-6.

9 Bonafide CP, Lin R, Zander M, et al. Association between exposure to nonactionable physiologic monitor alarms and response time in a children's Hospital. J Hosp Med 2015;10:345-51.

10 Paine CW, Goel VV, Ely E, et al. Systematic review of physiologic monitor alarm characteristics and pragmatic interventions to reduce alarm frequency. J Hosp Med 2016;11:136-44.

11 Winters BD, Cvach MM, Bonafide CP, et al. Technological distractions (Part 2): a summary of approaches to manage clinical alarms with intent to reduce alarm fatigue. Crit Care Med 2018;46:130-7.

12 Pater CM, Sosa TK, Boyer J, et al. Time series evaluation of improvement interventions to reduce alarm notifications in a paediatric Hospital. BMJ Qual Saf 2020;29:717-26.

13 Kobayashi L, Parchuri R, Gardiner FG, et al. Use of in situ simulation and human factors engineering to assess and improve emergency department clinical systems for timely telemetry-based detection of life-threatening arrhythmias. BMJ Qual Saf 2013;22:72-83.

14 Funk M, Ruppel H, Blake N, et al. Research: use of monitor watchers in hospitals: characteristics, training, and practices. Biomed Instrum Technol 2016;50:428-38.

15 Ruppel H, Funk M, Whittemore R, et al. Critical care nurses' clinical reasoning about physiologic monitor alarm customisation: an interpretive descriptive study. J Clin Nurs 2019;28:3033-41.

16 Schondelmeyer AC, Daraiseh NM, Allison B, et al. Nurse responses to physiologic monitor alarms on a general pediatric unit. J Hosp Med 2019;14:602-6.

17 Sowan AK, Tarriela AF, Gomez TM, et al. Nurses' perceptions and practices toward clinical alarms in a transplant cardiac intensive care unit: exploring key issues leading to alarm fatigue. JMIR Hum Factors 2015;2:e3.

18 Bonafide CP, Miller JM, Localio AR, et al. Association between mobile telephone interruptions and medication administration errors in a pediatric intensive care unit. JAMA Pediatr 2019. doi:10.1001/jamapediatrics.2019.5001. [Epub ahead of print: 20 Dec 2019].

19 Westbrook JI, Woods A, Rob MI, et al. Association of interruptions with an increased risk and severity of medication administration errors. Arch Intern Med 2010;170:683-90.

20 Sowan AK, Reed CC. A complex phenomenon in complex adaptive health care systems-alarm fatigue. JAMA Pediatr 2017;171:515-6. 